Article

\title{
Rapid Assessment of Functional Avidity of Tumor-Specific T Cell Receptors Using an Antigen-Presenting Tumor Cell Line Electroporated with Full-Length Tumor Antigen mRNA
}

\author{
Diana Campillo-Davo ${ }^{1, *}$, Maarten Versteven ${ }^{1}$ (D), Gils Roex ${ }^{1}{ }^{\mathbb{D}}$, Hans De Reu $^{1}$, \\ Sanne van der Heijden ${ }^{1}$, Sébastien Anguille 1,2,3, Zwi N. Berneman 1,2,3, \\ Viggo F. I. Van Tendeloo ${ }^{1}$ (D) and Eva Lion ${ }^{1,3, *}$ \\ 1 Tumor Immunology Group, Laboratory of Experimental Hematology, Vaccine \& Infectious Disease \\ Institute (VAXINFECTIO), Faculty of Medicine and Health Sciences, University of Antwerp, \\ 2610 Wilrijk, Antwerp, Belgium; maarten.versteven@uantwerpen.be (M.V.); \\ Gils.Roex@uantwerpen.be (G.R.); Hans.DeReu@uantwerpen.be (H.D.R.); \\ sanne@vdheijden.be (S.v.d.H.); sebastien.anguille@uantwerpen.be (S.A.); \\ zwi.berneman@uza.be (Z.N.B.); viggovantendeloo@gmail.com (V.F.I.V.T.) \\ 2 Division of Hematology, Antwerp University Hospital, 2650 Edegem, Antwerp, Belgium \\ 3 Center for Cell Therapy \& Regenerative Medicine, Antwerp University Hospital, \\ 2650 Edegem, Antwerp, Belgium \\ * Correspondence: Diana.CampilloDavo@uantwerpen.be (D.C.-D.); eva.lion@uantwerpen.be (E.L.); \\ Tel.: +32-3821-5254 (D.C.-D.); +32-3821-4112 (E.L.)
}

Received: 21 December 2019; Accepted: 15 January 2020; Published: 21 January 2020

\begin{abstract}
The functional avidity of T-cell receptor (TCR)-engineered T cells towards their cognate epitope plays a crucial role in successfully targeting and killing tumor cells expressing the tumorassociated antigen (TAA). When evaluating in vitro functional T-cell avidity, an important aspect that is often neglected is the antigen-presenting cell (APC) used in the assay. Cell-based models for antigen-presentation, such as tumor cell lines, represent a valid alternative to autologous APCs due to their availability, off-the-shelf capabilities, and the broad range of possibilities for modification via DNA or messenger RNA (mRNA) transfection. To find a valuable model APC for in vitro validation of TAA Wilms' tumor 1 (WT1)-specific TCRs, we tested four different WT1 peptide-pulsed HLA-A2+ tumor cell lines commonly used in T-cell stimulation assays. We found the multiple myeloma cell line U266 to be a suitable model APC to evaluate differences in mean functional avidity (EC50) values of transgenic TCRs following transfection in 2D3 Jurkat T cells. Next, to assess the dose-dependent antigen-specific responsiveness of WT1 TCR-engineered 2D3 T cells to endogenously processed epitopes, we electroporated U266 cells with different amounts of full-length antigen WT1 mRNA. Finally, we analyzed the functional avidity of WT1 TCR-transfected primary CD8 T cells towards WT1 mRNA-electroporated U266 cells. In this study, we demonstrate that both the APC and the antigen loading method (peptide pulsing versus full-length mRNA transfection) to analyze T-cell functional avidity have a significant impact on the EC50 values of a given TCR. For rapid assessment of the functional avidity of a cloned TCR towards its endogenously processed MHC I-restricted epitope, we showcase that the TAA mRNA-transfected U266 cell line is a suitable and versatile model APC.
\end{abstract}

Keywords: model antigen-presenting cell; T-cell functional avidity; full-length antigen mRNA electroporation; Wilms' tumor 1; cancer immunotherapy 


\section{Introduction}

T-cell receptor (TCR) gene therapy is a promising strategy in cancer immunotherapy, capitalizing on the use of TCR-engineered T cells targeting tumor-associated antigens (TAAs) expressed by cancer cells [1]. An essential element for the success of this type of therapy is the ability of TCR-engineered $\mathrm{T}$ cells to recognize the TAA, even at low epitope densities [2,3]. The threshold of activation of a $\mathrm{T}$ cell, defined as functional avidity, is a measurement of its effector response towards a particular surface density of the epitope [4]. Usually, it is evaluated in vitro by analyzing the response of $\mathrm{T}$ cells in peptide titration experiments. In this type of experiments, target cells are pulsed with decreasing concentrations of major histocompatibility complex (MHC)-binding peptides. The mean functional avidity, usually described by EC50, represents the peptide dose at which half-maximal activation of the T-cell population is reached. This value depends on the affinity and avidity of the TCR for its cognate peptide-MHC (pMHC) ligand and, therefore, it varies between different T-cell clones or TCR-engineered T cells. Generally, higher functional avidities-i.e., lower EC50 values-are linked to the recognition of lower epitope densities on the surface of antigen-presenting cells (APCs), and, thus, to better responses towards those cells $[5,6]$. Hence, the analysis of antigen-specific T-cell responses is vital at a clinical and research level to obtain the best TCRs for adoptive T-cell therapies $[7,8]$.

Measurement of T-cell functional avidity, however, can be challenging due to the vast array of analytical methods and the use of different types of cells presenting the antigen. Assays for the measurement of in vitro antigen-specific T-cell functional activity include direct cytotoxicity analysis by chromium $\left({ }^{51} \mathrm{Cr}\right)$ release [9] or flow cytometry-based killing assays [10], detection of intracellular expression of cytokines such as interferon-gamma (IFN- $\gamma$ ) or interleukin-2 (IL-2) [11,12], IFN- $\gamma$ or granzyme B enzyme-linked immunospot (ELISpot) assays [13] and enzyme-linked immunosorbent (ELISA) assays [14], mobilization of CD107a [15,16], and upregulation of activation markers, e.g., CD69 or CD137 [17]. In some models using TCR-deficient Jurkat cells, TCR activation is measured by the TCR-triggered expression of the green fluorescent protein (GFP) [18] or a combination of fluorescent proteins for the analysis of different transcription factors associated with TCR signaling [19]. Despite the multiple ways to analyze T-cell functional avidity, little is known about the impact that an APC may have on the result. This is important because T-cell activity may vary depending on the epitope density displayed by the APC, but also on the ability of an APC to promote T-cell activation. Among the multiple possibilities, cells of autologous origin, such as peripheral blood mononuclear cells (PBMCs), monocyte-derived dendritic cells (DCs) and B-lymphoblastoid cell lines represent the most common APCs in T-cell activation assays. Non-autologous cell-based models of APCs, including tumor cell lines such as T2 or K562 cells, are an alternative to the costly and laborious production of autologous APCs [20]. They represent an off-the-shelf approach that can be easily maintained, readily available, and modified as per request. Moreover, model APCs can be engineered with plasmid vectors or messenger RNAs (mRNAs) that encode the tumor antigens of interest. In particular, electroporation of antigen-encoding mRNA is a rapid and efficient method to induce neo-expression of the antigen in APCs. This technique allows the induction of multi-epitope T-cell responses, for example, in cancer patients following therapeutic vaccination with antigen-loaded DCs, such as the Wilms' tumor 1 (WT1) protein $[7,21]$. WT1 is a transcription factor overexpressed in leukemia and many solid tumors, but also present in healthy tissues [22]. Unfortunately, as an auto-antigen, T cells targeting self-TAAs such as WT1 with high avidity are scarce due to the negative selection that occurs in the thymus [23].

In the context of WT1-targeted adoptive TCR-engineered T-cell immunotherapies, methods to correctly asses the functional avidity of T cells engineered with WT1-specific TCRs are crucial for their success. In this study, we aimed to develop a reliable APC model for the evaluation of endogenously processed WT1 peptides and the avidity of WT1-specific TCRs. We analyzed the use of the HLA*A2:01-positive multiple myeloma cell line U266 as a tool for the rapid assessment of HLA-A2-restricted WT1-specific T-cell responses following electroporation with full-length WT1 mRNA, in comparison with WT1 peptide loading. To the best of our knowledge, this is the first study comparing exogenous peptide-loading and 
full-length antigen mRNA electroporation of target cells to study the functional avidity of epitope-specific TCR-redirected T cells.

\section{Results}

\subsection{Quantitation of WT1-Presenting Potential Odel APC}

To evaluate the capacity of different cell lines to be used as model APCs for presentation of WT1-derived epitopes by HLA-A2, the expression of surface HLA-A2 and natural intracellular WT1 proteins of four potential cell lines was quantified: T2 [24], U266 [25], K562-A2 [26] and Raji-A2 [27] cells (Figure 1). All cell lines expressed HLA-A2, with percentages ranging from $95 \%$ to $99 \%$ of HLAA2-positive cells (Figure 1, upper panel). With regards to the number of HLA-A2 molecules per cell, denoted as delta median fluorescence intensity (dMFI), T2 cells expressed the lowest levels of HLA-A2 molecules. On the contrary, Raji-A2 showed the highest levels of expression, whereas U266 and K562-A2 cells showed similar intermediate levels. Confirming literature, K562-A2 was the only cell line that clearly expressed WT1 $\left(68.14 \% \mathrm{WT} 1^{+}\right)$, whereas T2 and Raji-A2 cells expressed moderate amounts of the antigen $\left(15.79 \%\right.$ and $33.4 \% \mathrm{WT} 1^{+}$, respectively) and $\mathrm{U} 266$ cells the lowest amounts $\left(4.71 \% \mathrm{WT}^{+}\right)$ (Figure 1, lower panel).
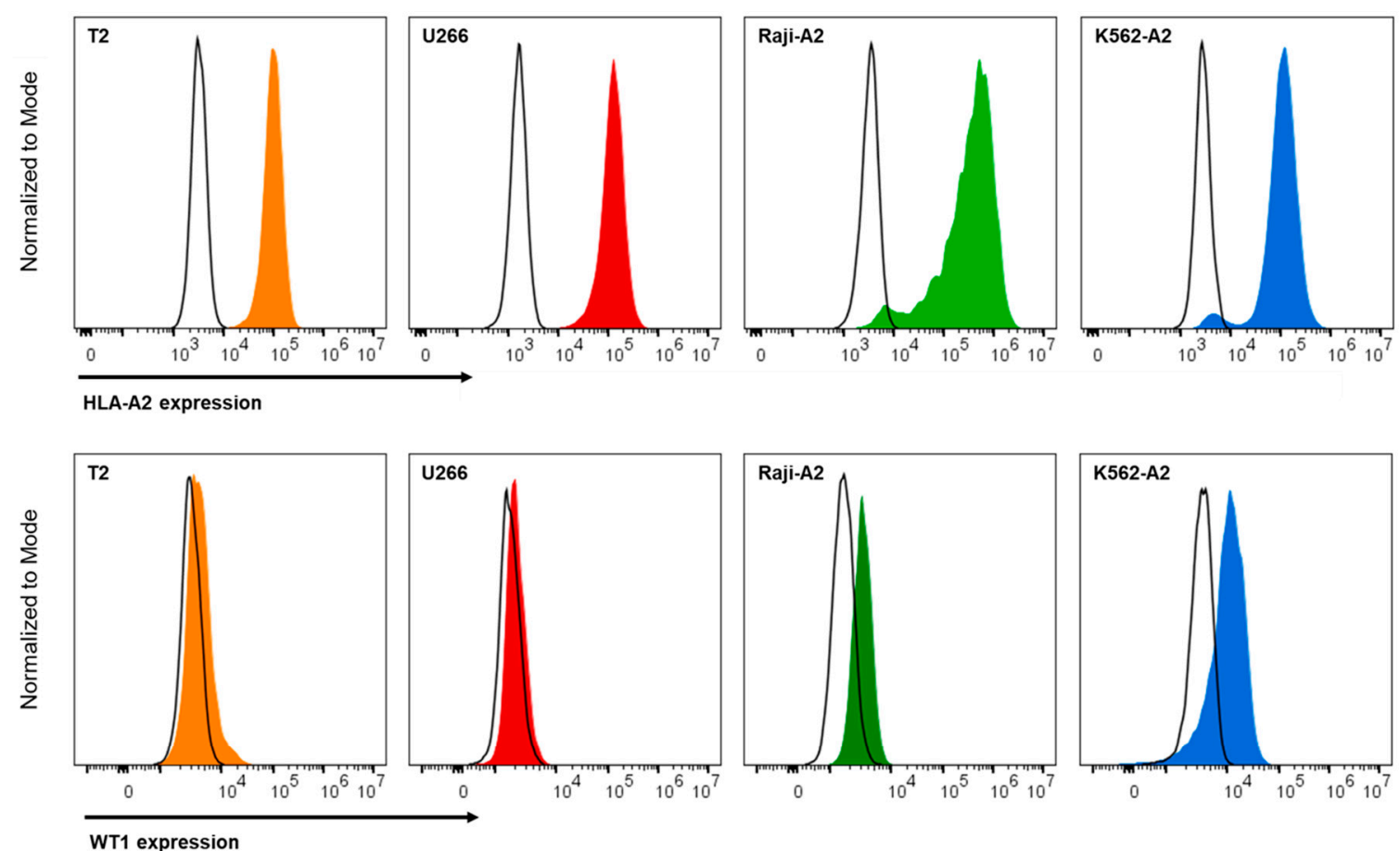

WT1 expression

\begin{tabular}{|c|c|c|c|}
\hline & $\%$ HLA-A2 $^{+}$cells & dMFI HLA-A2 & $\%$ WT1+ cells \\
\hline T2 & 99.66 & 92434 & 15.79 \\
\hline U266 & 99.64 & 120091 & 4.71 \\
\hline Raji-A2 & 96.25 & 377216 & 33.4 \\
\hline K562-A2 & 95.59 & 105297 & 68.14 \\
\hline
\end{tabular}

Figure 1. HLA-A2 and WT1 expression on four model antigen-presenting cell (APC) lines. Histograms (relative to mode) show the surface expression of HLA-A2 (upper panel) and the intracellular expression of WT1 (lower panel) of T2 (orange), U266 (red), Raji-A2 (green), and K562-A2 (blue) cell lines. HLA-A2 or WT1 expression (filled histograms) and isotype control (black line). The table shows HLA-A2 delta median fluorescence intensity (dMFI) values and percentage of HLA-A2 positive cells minus isotype staining (upper histograms) or percentages of WT1 positive cells minus isotype staining (lower histograms) for each cell line. HLA-A2, human leukocyte antigen A*02:01; WT1, Wilms' tumor 1 protein. 


\subsection{Functional Avidity of WT1-Specific T Cells Drastically Differs Depending on the APC Used}

To analyze the WT1 peptide-presenting capacity of the four model APC candidates, we used an in-house developed T-cell model assay, based on TCR-deficient $\mathrm{CD} 8^{+}$Jurkat 2D3 cells that are electroporated with TCR $\alpha \beta$-encoding mRNAs and express enhanced green fluorescent protein (EGFP) via nuclear factor of activated T cells (NFAT) upon antigen-specific TCR triggering [28,29]. Transgenic TCR expression for two HLA-A2-restricted TCRs directed against two epitopes of the WT1 protein, WT1 $1_{37-45}$ and WT1 $1_{126-134}$ (WT1.37 and WT1.126 TCR, respectively), was maximal for both TCRs $24 \mathrm{~h}$ after electroporation $\left(92.75 \pm 1.5 \% \mathrm{WT} 1.37 \mathrm{TCR}^{+}\right.$and $94.48 \pm 0.67 \% \mathrm{WT} 1.126 \mathrm{TCR}^{+} 2 \mathrm{D} 3$ cells; Supplementary Figure S1A). Pulsed with decreasing concentrations of WT1 $1_{37-45}$ or $W T 1_{126-134}$ peptides, the four model APCs were cultured with their respective WT1 TCR mRNA-electroporated 2D3 cells (Figure 2). The peak values of EGFP expression in 2D3 cells, corresponding to maximal T-cell activation, were detected with the highest peptide concentration for all cell lines (Figure 2A,B). The intensity of the T-cell response differed for both WT1-specific TCRs and depended on the APC type. When cultured with peptide-pulsed T2 cells, the highest percentages of EGFP ${ }^{+}$2D3 cells were reached as compared to U266 cells, Raji-A2, and K562-A2 cells, the latter promoting the poorest T-cell activation against both WT1 peptides. T2 cells, together with Raji-A2, displayed higher background levels of non-specific activation for both WT1.37 and WT1.126 TCR-electroporated 2D3 cells. Compared to the response observed with non-pulsed model APCs, the threshold of activation with T2 cells was reached at $10^{-9} \mathrm{M}$ for WT1.37 peptide ( $p=0.0002$; Figure 2A) and 10 $0^{-7} \mathrm{M}$ for WT1.126 ( $p=0.0001$; Figure 2B). In the case of $\mathrm{U} 266$, significant differences were detected at $10^{-7} \mathrm{M}$ for both peptides $(p=0.0007$ and 0.0456, respectively). As for Raji-A2 cells, the threshold of activation was reached at $10^{-8} \mathrm{M}$ for WT1.37 peptide $(p=0.0017)$ and $10^{-6} \mathrm{M}$ for WT1.126 $(p=0.0015)$. WT1.37 and WT1.126 TCR ${ }^{+} 2 \mathrm{D} 3$ cells were only able to significantly respond to K562-A2 cells pulsed with a concentration of $10^{-5} \mathrm{M}$ for both WT1 peptides ( $p=0.0284$, and $p=0.0012$, respectively). Uniformly comparing all cell lines, percentages of EGFP expression were normalized for the calculation of EC50 values (Figure 2C,D). In the same line, the EC50 values strongly varied between cell lines. Again, T2 cells were capable of promoting the best T-cell response for both WT1-specific TCRs (EC50: $1.06 \mathrm{nM}$ for WT1.37 TCR and $44.29 \mathrm{nM}$ for WT1.126 TCR). On the opposite side, peptide-pulsed K562-A2 cells induced T-cell responses at higher concentrations (EC50: $247.3 \mathrm{nM}$ for WT1.37 TCR and $1060 \mathrm{nM}$ for WT1.126 TCR). In the middle range, U266 and Raji-A2 cells promoted half-maximal responses at similar concentrations for the WT1.37 TCR (EC50 U266: 19.6 nM; EC50 Raji-A2: 10.08 nM), and WT1.126 TCR (EC50 U266: 148.8 nM; EC50 Raji-A2: $272 \mathrm{nM}$ ). These results show that for the same T-cell population expressing an antigen-specific TCR, the APC chosen for the peptide titration experiments has a pivotal role in the thresholds of T-cell activation.

\subsection{Mimicking Endogenous WT1 Expression}

Developing a model that can mimic the endogenous processing of WT1 in tumor cells in a controlled manner, among the four cell lines analyzed, T2 and K562-A2 cells are not model candidates because the former are unable to present internally-processed peptides and the latter are intrinsically highly positive for WT1. U266 and Raji-A2 cells generated similar EC50 values; however, they differed in natural WT1 expression levels. We selected the U266 cell line as the candidate model for further analysis due to the lower percentage of WT1 positive cells. Therefore, U266 cells, which naturally express HLA-A2 and minimal levels of WT1, were electroporated with increasing amounts of WT1 mRNA as the best model for presentation of internally-processed WT1 peptides (Figure 3). The increment in mRNA load resulted in an increase in the percentage of cells expressing the protein (Figure 3A), reaching the highest value of $\mathrm{WT}^{+} \mathrm{U} 266$ cells $(76.5 \pm 3.66 \%)$ upon electroporation of $20 \mu \mathrm{g}$ of WT1 mRNA. A significant difference between the $5 \mu \mathrm{g}$ and $20 \mu \mathrm{g}$ mRNA condition in \% of WT1-expressing cells was observed $(p=0.0078)$, demonstrating a dose-response dependency. Likewise, WT1 protein expression per cell increased with increasing mRNA concentrations after electroporation (Figure 3B). Next, we assessed the antigen-presenting capacity of the WT1 mRNA-electroporated U266 
cells in combination with WT1 TCR mRNA-electroporated 2D3 cells. EGFP expression by WT1.37 $\mathrm{TCR}^{+} 2 \mathrm{D} 3$ cells (Figure 3C, triangles) was significantly higher than mock electroporation $(0 \mu \mathrm{g}$ WT1 mRNA) when using $10 \mu \mathrm{g}(13.56 \pm 2.15 \% ; p=0.0348)$ and $20 \mu \mathrm{g}(18.48 \pm 3.28 \% ; p=0.0025)$ WT1 mRNA, but not with $5 \mu \mathrm{g}(9.04 \pm 2.28 \% ; p=0.3245)$. This indicates that WT1.37 epitope density on U266 cells after electroporation with $5 \mu \mathrm{g}$ of $W T 1 \mathrm{mRNA} / 5 \times 10^{6}$ cells per electroporation is not enough to surpass the threshold for WT1.37 TCR activation. On the other hand, WT1.126 TCR ${ }^{+}$2D3 cells (Figure 3C, circles) were not able to respond significantly to any of the amounts of WT1 mRNA used. Analyzing the amount of mRNA at which 50\% of maximal EGFP response was obtained, WT1.37 TCR-engineered T cells showed an EC50 value at $6.54 \mu \mathrm{g}$ WT1 mRNA-electroporated U266 (Figure 3D). This information could support indicating the minimum dosage of mRNA that should be used in APCs for the evaluation of specific T-cell clones or TCR-engineered T cells.

- T2 O U266 ○ Raji-A2 O K562-A2

A

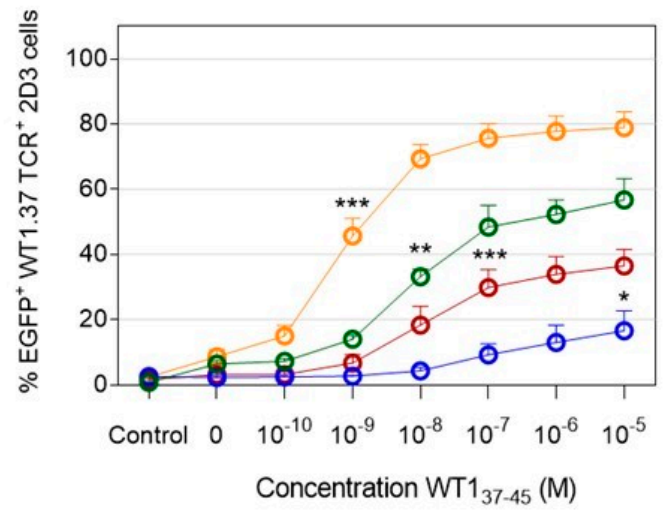

B

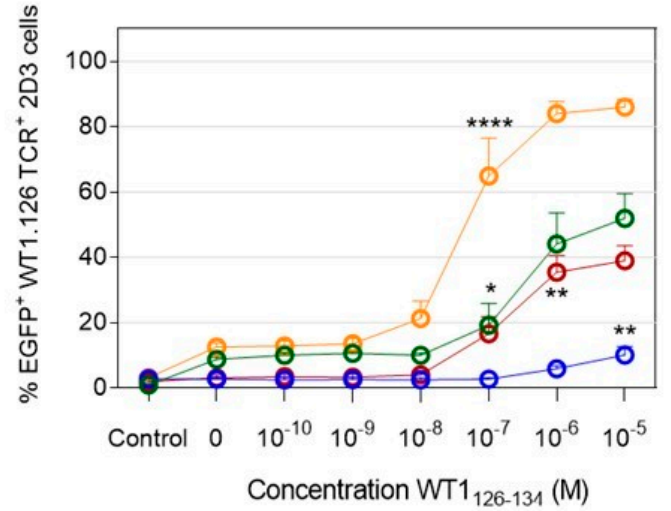

- T2 Ө U266 ค Raji-A2

K562-A2

C

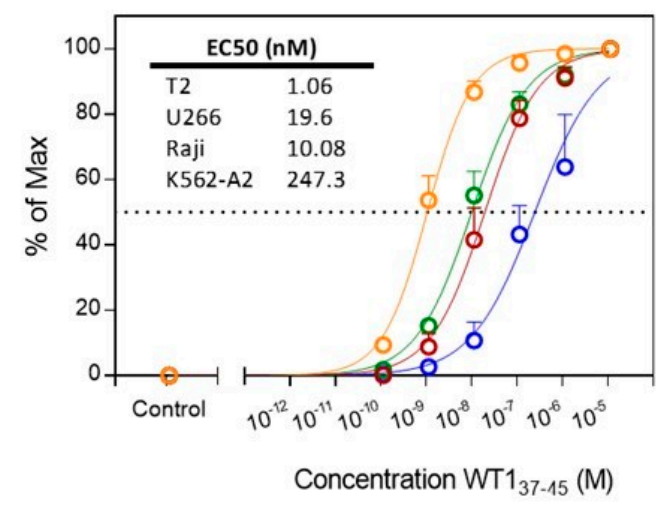

D

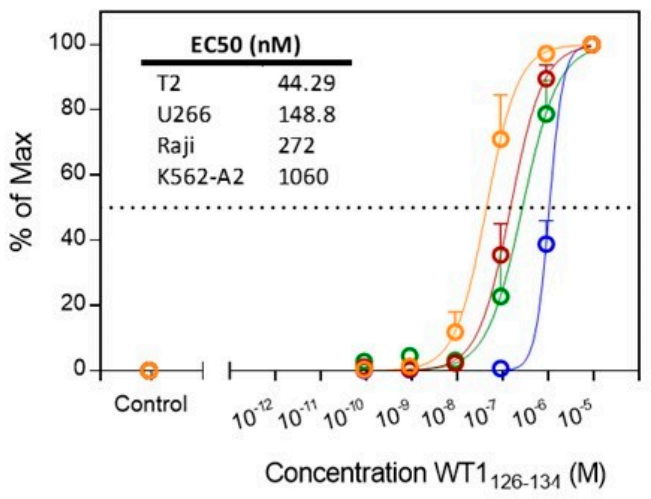

Figure 2. Epitope-specific T-cell activation by four model APC lines. Epitope-specific TCR activation was measured by expression of EGFP after WT1 $1_{37-45}(\mathbf{A}, \mathbf{C})$ or $\mathrm{WT}_{126-134}$ (B,D) peptide-specific TCR-transfected 2D3 cells were cultured for 18-22 h with model APCs T2, U266, Raji-A2 or K562-A2 cells that were pulsed with decreasing concentrations of WT1 peptide. Control depicts unstimulated 2D3 cells only. Graphs show the results of three to five independent replicates, showing $(\mathbf{A}, \mathbf{B})$ mean $\%$ $( \pm$ SEM) of EGFP positive cells and $(\mathbf{C}, \mathbf{D}) \%$ of maximal EGFP expression $( \pm$ SEM). $(\mathbf{A}, \mathbf{B})$ Data were analyzed using one-way ANOVA followed by Dunnett's post hoc test (comparing to non-peptide pulsed cells). EC50, the concentration of WT1 peptide at which $50 \%$ of the maximal EGFP expression is reached. ${ }^{*}, p<0.05 ; * *, p<0.01 ;{ }^{* *}, p<0.001 ;$ and ${ }^{* * *}, p<0.0001$. 
A

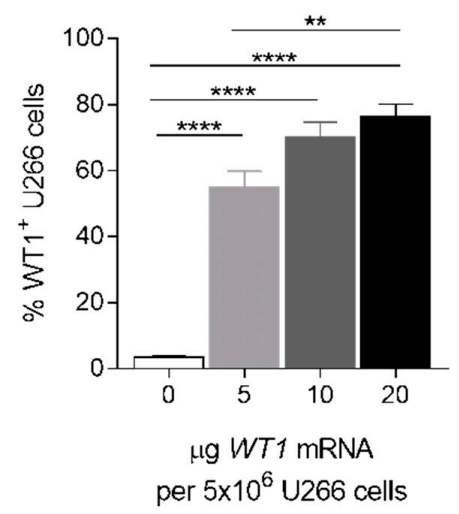

C

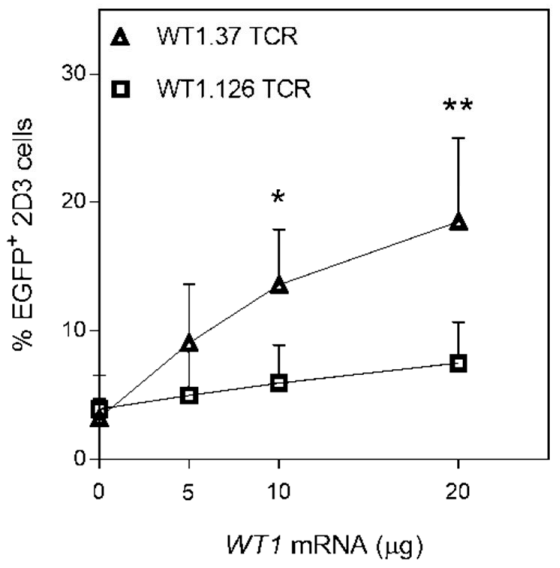

B

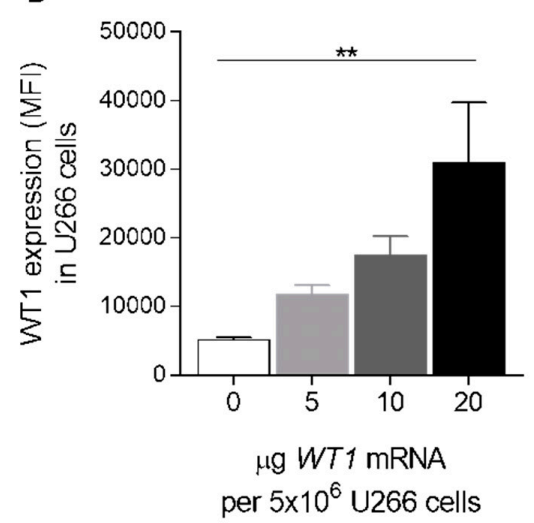

D

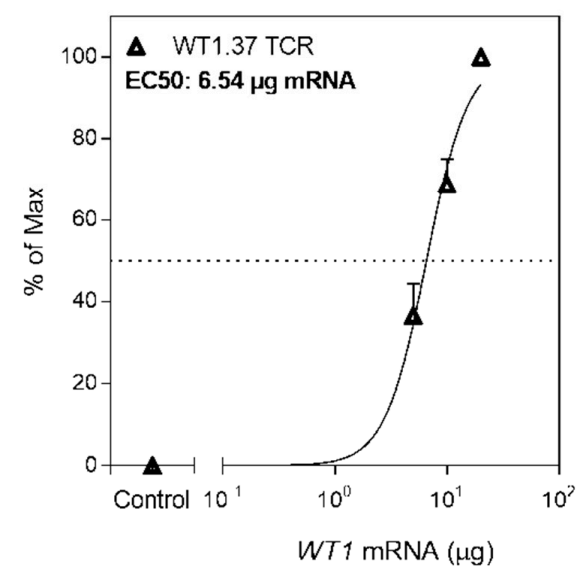

Figure 3. Epitope-specific TCR-engineered 2D3 cells can recognize full-length antigen WT1 mRNAelectroporated U266 cells in a dose-dependent manner. (A,B) Intracellular expression of WT1 is shown for U266 cells $24 \mathrm{~h}$ after electroporation with increasing amounts of WT1 mRNA per $5 \times 10^{6} \mathrm{U} 266$ cells. (C,D) 2D3 cells were electroporated with WT1 $1_{37-45^{-}}$or WT1 $1_{126-134}$-specific TCR mRNAs. Specific activation was detected by NFAT-promoted EGFP expression in 2D3 cells after 18-22 $\mathrm{h}$ co-culture with U266 cells electroporated with increasing amounts of WT1 mRNA. Graphs show the mean percentage of WT1 ${ }^{+}$U266 cells \pm SEM (A), the median fluorescence intensity (MFI) of U266 for WT1 expression \pm SEM (B), the percentage of maximal EGFP expression \pm SEM (C) and EC50, the amount of WT1 mRNA at which $50 \%$ of the maximal EGFP expression is reached (D) of 3-4 independent replicates. $(\mathbf{A}, \mathbf{B})$ One-way ANOVA followed by Tukey's post hoc test. (C) One-way ANOVA followed by Dunnett's post hoc test (comparing to mock-electroporated cells). ${ }^{*}, p<0.05 ;{ }^{* *}, p<0.01$; and ${ }^{* * * *}, p<0.0001$.

2.4. WT1 mRNA-Electroporated U266 Cells Activate WT1-Specific TCR-Redirected Primary Human CD8 T Cells in a Dose-Dependent Manner

Further evaluating the antigen-presenting capacity of U266 cells, the functional avidity of unstimulated primary human CD8 T cells was analyzed in the context of WT1.37 and WT1.126 peptides using our in-house developed double sequential electroporation (DSE) T cell assay [28]. In brief, purified CD8 T cells were subjected to DsiRNA-TCR mRNA to downregulate the expression of endogenous TCR, before codon-optimized WT1-specific TCR mRNA electroporation. For both WT1.37 and WT1.126 TCRs, high TCR expression was achieved $24 \mathrm{~h}$ after TCR mRNA electroporation $\left(66.9 \pm 5.345 \%\right.$ WT1.37/HLA-A2 tetramer ${ }^{+}$and $72.4 \pm 3.88 \%$ WT1.126/HLA-A2 tetramer ${ }^{+}$for eight donors; Supplementary Figure S1B). These WT1 TCR-engineered CD8 T cells were co-cultured with peptide-pulsed or WT1 mRNA-electroporated U266 and analyzed for WT1-specific CD8 T-cell activation and functional avidity by upregulation of CD69 and CD137 activation markers (Figure 4). For the WT1.37 peptide (Figure 4A), significant differences compared to the non-peptide pulsed U266 cells were still detected at a peptide concentration of $10^{-8} \mathrm{M}\left(8.61 \pm 1.07 \% \mathrm{CD} 69 / \mathrm{CD} 137^{+} ; p=0.0313\right)$, 
whereas the signal was lost at $10^{-9} \mathrm{M}\left(4.4 \pm 0.44 \% \mathrm{CD} 69 / \mathrm{CD} 137^{+} ; p=0.9931\right)$. In analogy with 2D3 cells, primary CD8 T cells electroporated with WT1.126 TCR were less sensitive to lower concentrations of the cognate peptide, compared to WT1.37 TCR ${ }^{+}$CD8 T cells. U266 cells pulsed with a WT1.126 peptide concentration of minimal $10^{-7}$ M elicited significant primary T-cell activation $(11.59 \pm 1.64 \%$ CD69/CD137 $; p=0.0010)$. EC50 values of functional avidity for WT1.37 (32.02 nM) and WT1.126 (135.3 nM) TCR-engineered primary CD8 T cells (Figure 4B) were comparable to those obtained for 2D3 cells ( $19.6 \mathrm{nM}$ and $148.8 \mathrm{nM}$, respectively). These findings confirm the usefulness of U266 cells in peptide-pulsing assays for the assessment of the functional avidity of primary TCR-redirected T cells. With regard to the WT1 mRNA-electroporated U266 cells (Figure 4C), only WT1.37 TCR-engineered primary CD8 T cells significantly responded to $10 \mu \mathrm{g}\left(8.92 \pm 1.71 \% \mathrm{CD} 69 / \mathrm{CD} 137^{+} ; p=0.0383\right)$ and $20 \mu \mathrm{g}$ of WT1 mRNA $\left(9.96 \pm 1.82 \% \mathrm{CD}^{2} / \mathrm{CD} 137^{+} ; p=0.0119\right)$. No significant differences with WT1.126 TCR-engineered CD8 T cells towards U266 cells electroporated with increasing amounts of electroporated WT1 mRNA were observed. In the case of WT1.37 TCR ${ }^{+}$CD8 T cells, $6.11 \mu \mathrm{g}$ of WT1 mRNA would be needed to reach upregulation of CD69 and CD137 in half of the maximal percentage of cells (Figure 4D), which is in line with the results using the 2D3 cell line (6.54 $\mu \mathrm{g}$ for WT1.37 TCR ${ }^{+}$; Figure 3D). Taken together, these findings show that evaluation of T-cell functional avidity with WT1 peptide-pulsed or WT1 mRNA-electroporated U266 cells remains constant for the TCRs analyzed regardless of the source of $\mathrm{T}$ cells, and that this system can help to distinguish TCRs that will respond to epitope densities of naturally processed WT1 protein. Hence, the application of U266 cells as a suitable APC model for WT1 antigen-specific T-cell assays.

A

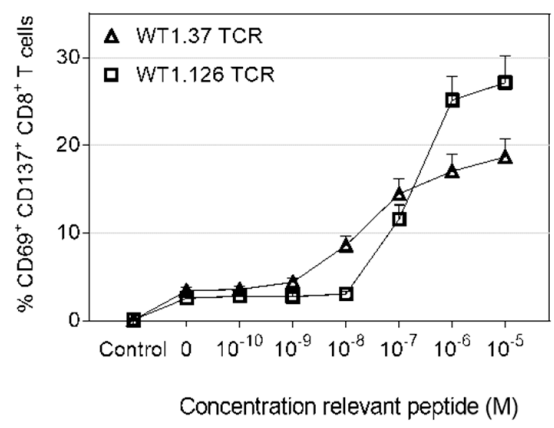

C

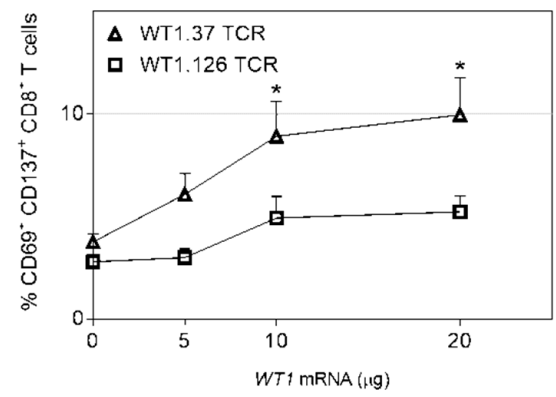

B

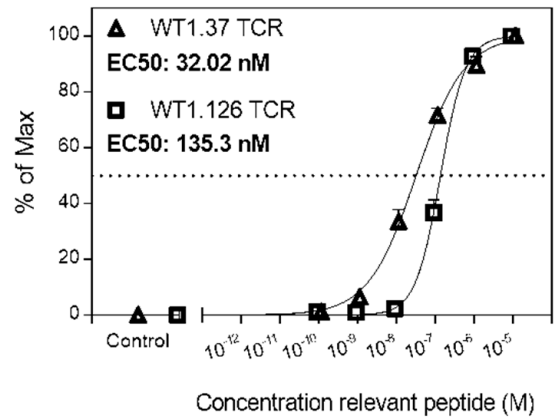

D

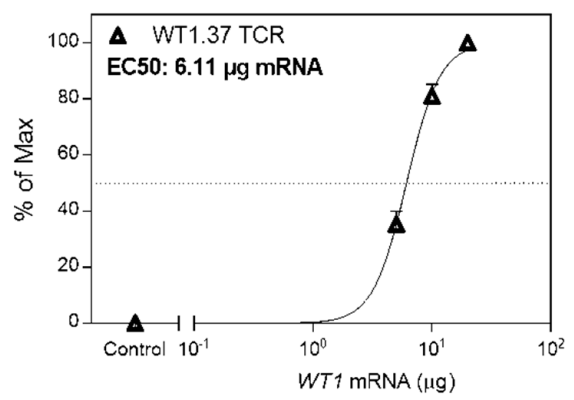

Figure 4. Analysis of functional avidity of WT1 epitope-specific primary CD8 T cells using WT1 peptide-pulsed and WT1 mRNA-electroporated U266 cells. Surface expression of both CD69 and CD137 activation markers was measured on WT1.37 (triangles) and WT1.126 (squares) peptide-specific DSE-engineered primary CD8 T cells $24 \mathrm{~h}$ after co-culture with U266 cells that were either pulsed with decreasing $(\mathbf{A}, \mathbf{B})$ concentrations of $\mathrm{WT1}_{37-45}$ or $\mathrm{WT1}_{126-134}$ peptide, or WT1 mRNA-electroporated (C,D). T cells only condition was used as a control. Graphs show mean \pm SEM of \% CD69/CD137 double positive CD8 T cells $(A, C)$ or $\%$ of maximal CD69/CD137 expression \pm SEM (B,D) for 6-8 donors. EC50, the concentration of WT1 peptides or amounts of electroporated WT1 mRNA at which 50\% of the maximal upregulation of CD69 and CD137 activation markers is reached. (A,C) One-way ANOVA followed by Dunnett's post hoc test (comparing to non-peptide pulsed or mock-electroporated cells). $*, p<0.05$. 


\section{Discussion}

Cell-based model APCs represent a valid alternative to autologous APCs and commonly used methods for analyzing antigen-specific T-cell activation status, for promoting ex-vivo T-cell expansion, and for the immunomonitoring of T-cell responses in the course of a viral infection or against cancer antigens in clinical trials [30]. To better understand the effect of the model APCs in the measurement of functional avidity of T cells, we compared four different model APC tumor cell lines (T2, U266, Raji-A2, and K562-A2). We showed a differential response in functional avidity of WT1-specific TCR-engineered $\mathrm{T}$ cells against different peptide-pulsed model APC tumor lines. This information is vital for an accurate calculation of T-cell responses when selecting T-cell clones or TCR-engineered T cells for cancer immunotherapy. Two of these cell lines, T2 and K562-A2, are routinely used in T-cell assays. In particular, T2 cells are widely used in peptide-MHC class I binding assays [31] due to their deficiency in transporter associated with antigen presentation (TAP). This complex is involved in the translocation of proteasome-processed peptides from the cytosol into the lumen of the ER [32]. The TAP deficiency in T2 cells results in MHC instability and reduction of nearly 70\% of HLA-A2 surface expression [33] that would explain the lower dMFI for HLA-A2 in these cells. The absence of TAP proteins also prevents the internal loading of TAP-dependent peptides onto the MHC molecules, making the HLA-A2 proteins available for the addition of exogenous peptides. Since endogenously processed and exogenously added peptides in peptide pulsing assays compete for the HLA-A2 molecules available [34], it is not surprising that TAP-deficient T2 cells outperformed Raji-A2, U266 cells, and K562-A2. However, manifested by the very low threshold of functional avidity when using peptide-pulsed T2 cells, they may reflect a non-physiological model that does not represent the actual T-cell functionality. This fact could lead to an overestimation of the T-cell functional avidity and to the selection of T-cell clones or TCRs that are of lower avidity towards more natural peptide-presenting target cells, particularly when screening for high avidity T-cell clones able to recognize tumor cells endogenously expressing, processing and presenting relevant tumor antigens.

A comparison between T2, K562-A2 and autologous B-LCL cells in a flow cytometry-based assay of T-cell killing capacity, showed that T cells cultured with peptide-pulsed T2 cells elicited a better response than those cultured with K562-A2 or B-LCL [35]. These results also indicate that T2 cells present a supraphysiological epitope density after incubation with exogenously added peptides. Interestingly, K562-A2 cells failed to properly activate T cells in our system. As reported by Britten et al., this cell line is a suitable model for interferon (IFN)- $\gamma$ ELISpot assays [26]. Britten and colleagues transduced K562-A2 cells with tyrosinase for its endogenous expression or exogenously pulsed them with tyrosinase-derived peptides. Therefore, T-cell responses to the natural expression of the ligand were not evaluated. Moreover, K562 cells naturally express WT1; thus, it does not represent a convenient model APC for the customization of WT1 mRNA intracellular levels. In this regard, Raji-A2 cells also did express the WT1 protein, albeit at very low levels. This fact, together with the dramatic overexpression of HLA-A2, tips the balance in favor of WT1-negative, naturally HLA-A2 expressing U266 cells.

In our study, the discrepancy in T-cell responses observed with the different cell lines highlights the importance of the APC when assessing functional avidity, but also the influence of the source of the studied epitope. In many types of malignancies, tumor cells downregulate the expression of MHC proteins [36] or have deficiencies in their antigen processing pathways [37], which negatively impacts the presentation and density of peptides on their surface. Since the expression of a precise pMHC complex on the surface of the model APC depends on its capability to internally process full antigens, the sole addition of exogenous synthetic peptides for T-cell assays may provide an incomplete and potentially misleading scenario for the analysis of T-cell functional avidity. We show that U266 cells can be efficiently electroporated with full-length antigen WT1 mRNA. The electroporation of higher amounts of mRNA was correlated with an increase in WT1 expression. This represents a flexible system in which different amounts of mRNA can be tested prior to clinical trials with full-length tumor antigen mRNA-electroporated DCs. Moreover, WT1 mRNA-electroporated U266 cells could be a useful alternative cell-based antigen presentation model to DCs [38,39], K562 cells [39] or PBMCs [40] 
for the oligo-clonal detection of WT1-specific T cell populations and immunomonitoring of T-cell responses in full-antigen mRNA-electroporation DC vaccination trials. The generation of autologous APCs for T-cell assays is not always possible and often entails a lengthy process required for every donor. This could be overcome by the use of U266 cells as model APCs. Moreover, epitope-specific T cell responses induced by WT1 mRNA-electroporated U266 cells can be compared to a peptide-titration curve using the same cell line. Another advantage of WT1 mRNA-electroporated U266 is the possibility of off-the-shelf production by freezing the cells after electroporation.

Our study also confirms the suitability of 2D3 cells for the analysis of TCR avidity, thanks to their expression of human CD8 co-receptor, the absence of a native TCR $\alpha \beta$, the simplicity to engineer them with an antigen-specific TCR, and the expression of EGFP upon TCR triggering. The lack of endogenous TCR eliminates the possibility of TCR mispairing between endogenous and transgenic TCRs [41]. Therefore, EGFP expression can be directly correlated with the degree of introduced TCR triggering, i.e., the capacity of different APCs to present a peptide and to activate T cells. These findings are in accordance with previous reports showing that tetramers allow the quantification of antigen-specific T cells, but do not always provide accurate data on the functionality of T cells [42-45]. Regarding primary human CD8 T cells, activation markers enable the identification of all responder $\mathrm{T}$ cells after TCR triggering. One of the most common activation markers in flow cytometric analysis is CD137. Combined with CD69, CD137 is a powerful and sensitive tool to measure epitope-specific T cells regardless of the T-cell state of differentiation or subset [46].

\section{Materials and Methods}

\subsection{Cell Lines and Primary Cells}

The TCR $\alpha \beta$-deficient, CD8 $\alpha \beta$ and NFAT-EGFP stably-transfected T cell acute leukemia 2D3 cell line $[28,29]$ was kindly provided by Prof. Haruo Sugiyama (Osaka University Graduate School of Medicine, Osaka, Japan) and maintained in Roswell Park Memorial Institute 1640 (RPMI) culture medium (Life Technologies, Merelbeke, Belgium) supplemented with 10\% FBS. The HLA-A*02:01-positive WT1-negative human transporter associated with antigen presentation (TAP)-deficient lymphoblastoid T2 cell line was kindly provided by Dr. Pierre Van der Bruggen (Ludwig Institute for Cancer Research, Brussels, Belgium). U266 is an HLA-A*02:01-positive, WT1-negative multiple myeloma cell line and was a kind gift from Dr. Wilfred T.V. Germeraad (GROW School for Oncology \& Developmental Biology, Maastricht University, Maastricht, The Netherlands). The HLA-A*02:01-transduced Burkitt's lymphoma Raji-derived Raji-A2 cell line was kindly provided by Dr. Mirjam Heemskerk (Leiden University Medical Center, Leiden, The Netherlands). The HLA-A*02:01-transduced human chronic myelogenous leukemia K562-derived K562-A2 cell line was a kind gift from Dr. Cedrik Britten (R\&D Oncology, GlaxoSmithKline, Stevenage, UK). T2, U266, Raji-A2, and K562-A2 cells were cultured in Iscove's Modified Dulbecco's Medium (IMDM; Life Technologies,) supplemented with 10\% FBS. All cell lines were maintained in a logarithmic growth phase at $37^{\circ} \mathrm{C}$ in a humidified atmosphere supplemented with $5 \% \mathrm{CO}_{2}$.

Blood samples of healthy anonymous donors were purchased from the Blood Service of the Flemish Red Cross (Mechelen, Belgium) following the approval by the Ethics Committee of the Antwerp University Hospital and the University of Antwerp (reference number 16/35/357). Peripheral blood mononuclear cells were isolated by density gradient centrifugation using Ficoll-Paque PLUS (GE Healthcare, Diegem, Belgium), and CD8 T cells were selected using human CD8 magnetic microbeads for magnetic-activated cell sorting (MACS) according to the manufacturer's instructions (Miltenyi Biotec, Leiden, The Netherlands). The purity of CD8 T cells after MACS isolation was analyzed using a CytoFLEX flow cytometer (Beckman Coulter, Suarlée, Belgium) after staining with FITC-labeled anti-CD8, PE-conjugated anti-CD4 and PerCP-conjugated anti-CD3 monoclonal antibodies (mAbs; Becton-Dickinson (BD) Biosciences, Erembodegem, Belgium). After MACS isolation, CD8 T cells were centrifuged and resuspended in cryopreservation medium consisting of fetal bovine serum (FBS; Life 
Technologies) supplemented with 10\% dimethyl sulfoxide (DMSO; Sigma-Aldrich, Diegem, Belgium). Aliquots of $20-35 \times 10^{6}$ cells $/ \mathrm{mL}$ were transferred to Mr. Frosty freezing containers (Thermo Fisher Scientific, Erembodegem, Belgium) filled with isopropyl alcohol (Yvsolab, Turnhout, Belgium) and kept in a $-80^{\circ} \mathrm{C}$ freezer for at least seven days up to three weeks. Aliquots were thawed in pre-warmed AIM-V (Life Technologies) supplemented with 10\% human AB serum (hAB; Life Technologies) and rested for at least one hour in a humidified $5 \% \mathrm{CO}_{2}$ incubator at $37^{\circ} \mathrm{C}$.

\subsection{In Vitro Transcription of $m R N A$}

The cloning of WT1-specific TCR genes, generation of the pST1 DNA plasmids containing the TCR constructs and generation of WT1-specific TCR mRNA by in vitro transcription (IVT) were performed as previously described [28,29]. Clinical-grade codon-optimized Sig-DC-LAMP WT1 mRNA encoding isoform D of WT1 [21] was purchased from eTheRNA immunotherapies (Niel, Belgium).

\subsection{Electroporation}

Electroporation of 2D3 cells with WT1-specific TCR mRNA was performed as previously described [28]. Double sequential electroporation (DSE) of human primary CD8 T cells was performed following [28], with minor modifications. Briefly, 10 or $20 \times 10^{6}$ thawed viable human primary CD8 T cells were resuspended in 200 or $400 \mu \mathrm{L}$ of serum-free Opti-MEM medium (Life Technologies) after thawing and transferred to a $4 \mathrm{~mm}$-gap electroporation cuvette (Cell Projects, Harrietsham, UK). Next, cells were electroporated with 16 or $32 \mu \mathrm{L}$ of a pool containing $100 \mu \mathrm{M}$ of TRAC- and TRBC-specific DsiRNAs (Integrated DNA Technologies) in a ratio of 1:1. After electroporation, cells were transferred to pre-warmed AIM-V medium supplemented with $10 \% \mathrm{hAB}$, rested at $37^{\circ} \mathrm{C}$ in a humidified atmosphere supplemented with $5 \% \mathrm{CO}_{2}$ for at least $20 \mathrm{~min}$, centrifuged $(300 \times \mathrm{g}, 3 \mathrm{~min})$, transferred to 6-well plates and then incubated for $24 \mathrm{~h}$. Second electroporation with in vitro transcribed mRNA was performed following the same protocol, using $1 \mu \mathrm{g}$ of mRNA per $10^{6}$ cells. For the electroporation of U266 cells, $5 \times 10^{6}$ viable cells were washed once with Opti-MEM I medium (Life Technologies), resuspended in $200 \mu \mathrm{L}$ of the same medium, and then transferred to $4 \mathrm{~mm}$-gap cuvettes (Cell Projects). Next, 5, 10 or $20 \mu \mathrm{g}$ of clinical-grade IVT WT1 mRNA was added to the cells before electroporation. Cells were electroporated in a Gene Pulser Xcell ${ }^{\mathrm{TM}}$ device (Bio-Rad Laboratories, Temse, Belgium) using the Time constant protocol ( $300 \mathrm{~V}, 8 \mathrm{~ms}$, one pulse). After electroporation, all cells were transferred to pre-warmed recovery medium (RPMI supplemented with 10\% FBS for 2D3 cells; AIM-V medium supplemented with $10 \% \mathrm{hAB}$ for human primary T cells, and IMDM supplemented with $10 \%$ FBS for $\mathrm{U} 266$ cells) and rested for at least $20 \mathrm{~min}$ in a humidified $5 \% \mathrm{CO}_{2}$ incubator at $37^{\circ} \mathrm{C}$. Before co-culture, cells were washed, resuspended in fresh medium and incubated for $4 \mathrm{~h}$. When necessary, cells were electroporated without mRNA (mock) as a negative control.

\subsection{Flow Cytometry}

HLA-A*02:01 positivity of T2, U266, Raji-A2, K562-A2 cells was analyzed by direct staining using a PE-conjugated anti-human HLA-A*02 antibody (clone BB7-2; BioLegend, London, UK). HLA-A*02:01 expression on PBMC samples was detected by incubation with the supernatant of the hybridoma BB7.2 cell line (producer of anti-HLA-A*02 antibody, ATCC) for $15 \mathrm{~min}$ at room temperature. Then, cells were washed with FACS buffer (FACSFlow sheath fluid (BD Biosciences), 0.1\% bovine serum albumin (BSA) (Sigma-Aldrich), 0.05\% sodium azide (Merck, Overijse, Belgium), labeled with FITC-conjugated polyclonal rabbit anti-mouse immunoglobulins (Dako, Heverlee, Belgium) for $15 \mathrm{~min}$ at room temperature protected from light. WT1 expression was analyzed in samples from T2, U266, Raji-A2, and K562-A2 cell lines or electroporated U266 cells $24 \mathrm{~h}$ after WT1 mRNA electroporation by intracellular staining. Cells were harvested for fixation and permeabilization using the Foxp3/Transcription factor staining buffer set (eBioscience, Life Technologies) according to the manufacturer's instructions. Next, cells were labeled with unconjugated mouse anti-human WT1 monoclonal antibody (clone 6F-H2, Dako)—which recognizes an epitope within residues 1-181 of all isoforms of the full-length WT1 protein-followed 
by PE-conjugated polyclonal rabbit anti-mouse immunoglobulins (Dako). As a control, samples were only incubated with PE-conjugated polyclonal rabbit anti-mouse immunoglobulins. WT1-specific TCR surface expression was evaluated $24 \mathrm{~h}$ after TCR mRNA electroporation in 2D3 and primary CD8 $\mathrm{T}$ cells. For 2D3 cells, samples were labeled with FITC-conjugated anti-CD8 (BD Biosciences) and PE-conjugated anti-pan TCR $\alpha \beta$ (Miltenyi Biotec) or isotype control mAb (BD Biosciences) for $15 \mathrm{~min}$ at room temperature. For primary CD8 T cells, samples were labeled with PE-conjugated WT1 $1_{37-45}$ or $\mathrm{WT}_{126-134}$ peptide/HLA-A*02:01 tetramers for $30 \mathrm{~min}$ at $37^{\circ} \mathrm{C}$ [28]. Then, cells were washed and labeled with FITC-conjugated anti-CD8 and PerCP-conjugated anti-CD3 mAbs (BD Biosciences) for 15 min at room temperature. All samples were washed previous to analysis on a CytoFLEX cytometer (Beckman Coulter).

\subsection{Peptide-Pulsing of Tumor Cells}

Viable T2, U266, Raji-A2, and K562-A2 cells were harvested, washed once in serum-free IMDM medium, and resuspended using the same medium at a final concentration of $10^{6}$ cells $/ \mathrm{mL}$. Cells were split in tubes and pulsed with WT1 $1_{37-45}$ (VLDFAPPGA) or WT1 $1_{126-134}$ peptide (RMFPNAPYL) (JPT Peptide Technologies, Berlin, Germany) at decreasing concentrations of a ten-fold serial dilution from a concentration of $10 \mu \mathrm{g} / \mathrm{mL}$ for $60 \mathrm{~min}$ at room temperature under constant motion. After incubation, cells were washed and resuspended in IMDM supplemented with $10 \%$ FBS at a concentration of $5 \times 10^{5}$ cells $/ \mathrm{mL}$.

\subsection{Co-Cultures}

Electroporated 2D3 or DSE primary CD8 T cells were co-cultured with peptide-pulsed T2, U266, Raji-A2 and K562-A2 cells or electroporated U266 cells in triplicate in 96-well round-bottom plates at an effector:target (E:T) ratio of 2:1 (2D3 cells) or 4:1 (primary CD8 T cells). 2D3 cells or primary CD8 T cells cultured alone served as negative controls. Co-cultures were incubated for $18-22 \mathrm{~h}$ at $37^{\circ} \mathrm{C}$ in a humidified atmosphere supplemented with $5 \% \mathrm{CO}_{2}$.

\subsection{Analysis of Epitope-Specific T-Cell Activation}

After co-culture, cells were harvested and analyzed for epitope-specific expression of the enhanced green fluorescent protein (EGFP; 2D3 cells) or expression of the activation markers CD137 and CD69 (primary CD8 T cells). Samples from 2D3 cell co-cultures were washed, incubated with PE-conjugated anti-CD8 for $15 \mathrm{~min}$ at room temperature. Then, samples were rewashed and stained with the nucleic acid dye 7-aminoactinomycin D (7-AAD; BD Biosciences) for $10 \mathrm{~min}$ at room temperature for the exclusion of nonviable cells before analysis on a CytoFLEX cytometer (Beckman Coulter). Samples from primary CD8 T cell co-cultures were washed and stained with anti-human PE-conjugated anti-CD137, PerCP-Cy5.5-conjugated anti-CD3, APC-Cy7-conjugated anti-CD69 (BD Biosciences) and Pacific Blue-conjugated anti-CD8 (Life Technologies) monoclonal antibodies and LIVE/DEAD fixable aqua dead cell stain kit (Thermo Fisher Scientific) for $15 \mathrm{~min}$ at room temperature. After incubation, cells were washed and analyzed using a FACSAria II cytometer (BD Biosciences).

\subsection{Statistical Analysis}

Data from flow cytometers were analyzed using FlowJo v10.2 software (TreeStar Inc, Ashland, OR, USA). Prism v5 software (GraphPad, San Diego, CA, USA) was used for graphing, statistical calculations and calculation of EC50 values. Data were analyzed using one-way analysis of variance (ANOVA) followed by Dunnett's or Tukey's post hoc test where applicable for multiple comparisons. Results were considered to be statistically significant when $p$-value was less than $0.05 .{ }^{*}$ indicates $p<0.05,{ }^{* *}$ indicates $p<0.01, * * *$ indicates $p<0.001$ and ${ }^{* * * *}$ indicates $p<0.0001$. 


\section{Conclusions}

Our study demonstrates the relevance of comparing the APCs used in T-cell assays and the influence they may have when evaluating T-cell functional avidity. Here, we provide a versatile model to evaluate HLA-A2-restricted WT1 epitope-specific responses by TCR-engineered T cells based on the combination of a tumor cell-based APC with a rapid engineering method such as mRNA electroporation. This model could be valuable for the screening and selection of WT1-specific high-avidity TCRs intended for TCR-engineered therapies without the need for primary APCs. It can potentially be used to analyze other TAA-specific T cells, in particular, for those $\mathrm{T}$ cells with low circulating levels that are reactive against tumor-associated autoantigens in the style of WT1. Eventually, this platform could provide the basis for the development of an immunomonitoring tool to evaluate TAA-specific T-cell activity in clinical trials using TAA mRNA-electroporated DC vaccines for cancer immunotherapy.

Supplementary Materials: The following are available online at http://www.mdpi.com/2072-6694/12/2/256/s1, Figure S1: WT1-specific TCR expression on 2D3 and primary CD8 T cells.

Author Contributions: Conceptualization, D.C.-D., V.F.I.V.T. and E.L.; Data curation, D.C.-D.; Formal analysis, D.C.-D.; Funding acquisition, Z.N.B., V.F.I.V.T. and E.L.; Investigation, D.C.-D., H.D.R. and S.v.d.H.; Methodology, D.C.-D.; Project administration, D.C.-D., Z.N.B., V.F.I.V.T. and E.L.; Resources, Z.N.B., V.F.I.V.T. and E.L.; Supervision, Z.N.B., V.F.I.V.T. and E.L.; Validation, D.C.-D.; Visualization, D.C.-D.; Writing-original draft, D.C.-D., V.F.I.V.T. and E.L.; Writing-review \& editing, D.C.-D., M.V., G.R., H.D.R, S.v.d.H., S.A., Z.N.B., V.F.I.V.T. and E.L. All authors have read and agreed to the published version of the manuscript.

Funding: This work was funded by grant FAF-C/2016/764 from the Belgian Foundation against Cancer (Stichting tegen Kanker), by grant G053518N from the Fund for Scientific Research Flanders (FWO Vlaanderen), and by grants from the Baillet-Latour and the Cellular therapy fund via the UZA Foundation and the Kaushik Bhansali Fund. D.C.-D. is supported by a DOC-PRO Ph.D. grant of the Special Research Fund (BOF) of the University of Antwerp. M.V. is funded by an SB-fellowship from the Research Foundation Flanders (FWO; grant number 1S24517N). G.R. is supported by an Emmanuel van der Schueren fellowship from Kom op tegen Kanker (Stand up to Cancer, Belgium) and the public utility foundation MeToYou (Belgium). S.A. is a Senior Clinical Investigator of the Research Fund (FWO)-Flanders (Belgium). E.L. is supported by funds of Stand up to Cancer (Kom op tegen Kanker). Part of this work was performed under the umbrella of the European COST TD1104 action (EP4Bio2MED; www.electroporation.net).

Acknowledgments: We thank David A. Price (Division of Infection and Immunity, Cardiff University School of Medicine, Cardiff, UK) for kindly providing the monomers for tetramer preparation.

Conflicts of Interest: The authors declare no conflict of interest. The funders had no role in the design of the study; in the collection, analyses, or interpretation of data; in the writing of the manuscript, or in the decision to publish the results.

\section{References}

1. Tendeiro Rego, R.; Morris, E.C.; Lowdell, M.W. T-cell receptor gene-modified cells: Past promises, present methodologies and future challenges. Cytotherapy 2019, 21, 341-357. [CrossRef]

2. Bullock, T.N.; Colella, T.A.; Engelhard, V.H. The density of peptides displayed by dendritic cells affects immune responses to human tyrosinase and gp100 in HLA-A2 transgenic mice. J. Immunol. 2000, 164, 2354-2361. [CrossRef] [PubMed]

3. Gonzalez, P.A.; Carreno, L.J.; Coombs, D.; Mora, J.E.; Palmieri, E.; Goldstein, B.; Nathenson, S.G.; Kalergis, A.M. $\mathrm{T}$ cell receptor binding kinetics required for $\mathrm{T}$ cell activation depend on the density of cognate ligand on the antigen-presenting cell. Proc. Natl. Acad. Sci. USA 2005, 102, 4824-4829. [CrossRef] [PubMed]

4. Allard, M.; Hebeisen, M.; Rufer, N. Assessing T Cell Receptor Affinity and Avidity Against Tumor Antigens. In Oncoimmunology: A Practical Guide for Cancer Immunotherapy; Zitvogel, L., Kroemer, G., Eds.; Springer International Publishing: Cham, Switzerland, 2018; pp. 665-679. [CrossRef]

5. Snyder, J.T.; Alexander-Miller, M.A.; Berzofskyl, J.A.; Belyakov, I.M. Molecular mechanisms and biological significance of CTL avidity. Curr. HIV Res. 2003, 1, 287-294. [CrossRef] [PubMed]

6. Fahmy, T.M.; Bieler, J.G.; Edidin, M.; Schneck, J.P. Increased TCR avidity after T cell activation: A mechanism for sensing low-density antigen. Immunity 2001, 14, 135-143. [CrossRef] 
7. Van Tendeloo, V.F.; Van de Velde, A.; Van Driessche, A.; Cools, N.; Anguille, S.; Ladell, K.; Gostick, E.; Vermeulen, K.; Pieters, K.; Nijs, G.; et al. Induction of complete and molecular remissions in acute myeloid leukemia by Wilms' tumor 1 antigen-targeted dendritic cell vaccination. Proc. Natl. Acad. Sci. USA 2010, 107, 13824-13829. [CrossRef]

8. Chapuis, A.G.; Ragnarsson, G.B.; Nguyen, H.N.; Chaney, C.N.; Pufnock, J.S.; Schmitt, T.M.; Duerkopp, N.; Roberts, I.M.; Pogosov, G.L.; Ho, W.Y.; et al. Transferred WT1-reactive CD8+ T cells can mediate antileukemic activity and persist in post-transplant patients. Sci. Transl. Med. 2013, 5, 174ra27. [CrossRef]

9. Kohrt, H.E.; Shu, C.T.; Stuge, T.B.; Holmes, S.P.; Weber, J.; Lee, P.P. Rapid assessment of recognition efficiency and functional capacity of antigen-specific T-cell responses. J. Immunother. 2005, 28, 297-305. [CrossRef]

10. Takagi, A.; Horiuchi, Y.; Matsui, M. Characterization of the flow cytometric assay for ex vivo monitoring of cytotoxicity mediated by antigen-specific cytotoxic T lymphocytes. Biochem. Biophys. Res. Commun. 2017, 492, 27-32. [CrossRef]

11. Lovelace, P.; Maecker, H.T. Multiparameter Intracellular Cytokine Staining. Methods Mol. Biol. 2018, 1678, 151-166. [CrossRef]

12. Schmidt, T.; Sester, M. Detection of antigen-specific T cells based on intracellular cytokine staining using flow-cytometry. Methods Mol. Biol. 2013, 1064, 267-274. [CrossRef]

13. Malyguine, A.M.; Strobl, S.; Dunham, K.; Shurin, M.R.; Sayers, T.J. ELISPOT Assay for Monitoring Cytotoxic T Lymphocytes (CTL) Activity in Cancer Vaccine Clinical Trials. Cells 2012, 1, 111-126. [CrossRef] [PubMed]

14. Li Pira, G.; Ivaldi, F.; Dentone, C.; Righi, E.; Del Bono, V.; Viscoli, C.; Koopman, G.; Manca, F. Evaluation of antigen-specific T-cell responses with a miniaturized and automated method. Clin. Vaccine Immunol. 2008, 15, 1811-1818. [CrossRef] [PubMed]

15. Rubio, V.; Stuge, T.B.; Singh, N.; Betts, M.R.; Weber, J.S.; Roederer, M.; Lee, P.P. Ex vivo identification, isolation and analysis of tumor-cytolytic T cells. Nat. Med. 2003, 9, 1377-1382. [CrossRef] [PubMed]

16. Betts, M.R.; Brenchley, J.M.; Price, D.A.; De Rosa, S.C.; Douek, D.C.; Roederer, M.; Koup, R.A. Sensitive and viable identification of antigen-specific CD8+ T cells by a flow cytometric assay for degranulation. J. Immunol. Methods 2003, 281, 65-78. [CrossRef]

17. Wolfl, M.; Kuball, J.; Ho, W.Y.; Nguyen, H.; Manley, T.J.; Bleakley, M.; Greenberg, P.D. Activation-induced expression of CD137 permits detection, isolation, and expansion of the full repertoire of CD8+ T cells responding to antigen without requiring knowledge of epitope specificities. Blood 2007, 110, 201-210. [CrossRef]

18. Morimoto, S.; Fujiki, F.; Kondo, K.; Nakajima, H.; Kobayashi, Y.; Inatome, M.; Aoyama, N.; Nishida, Y.; Tsuboi, A.; Oka, Y.; et al. Establishment of a novel platform cell line for efficient and precise evaluation of T cell receptor functional avidity. Oncotarget 2018, 9, 34132-34141. [CrossRef]

19. Rosskopf, S.; Leitner, J.; Paster, W.; Morton, L.T.; Hagedoorn, R.S.; Steinberger, P.; Heemskerk, M.H.M. A Jurkat 76 based triple parameter reporter system to evaluate TCR functions and adoptive T cell strategies. Oncotarget 2018, 9, 17608-17619. [CrossRef]

20. Eggermont, L.J.; Paulis, L.E.; Tel, J.; Figdor, C.G. Towards efficient cancer immunotherapy: Advances in developing artificial antigen-presenting cells. Trends Biotechnol. 2014, 32, 456-465. [CrossRef]

21. Anguille, S.; Van de Velde, A.L.; Smits, E.L.; Van Tendeloo, V.F.; Juliusson, G.; Cools, N.; Nijs, G.; Stein, B.; Lion, E.; Van Driessche, A.; et al. Dendritic cell vaccination as postremission treatment to prevent or delay relapse in acute myeloid leukemia. Blood 2017, 130, 1713-1721. [CrossRef]

22. Yang, L.; Han, Y.; Suarez Saiz, F.; Minden, M.D. A tumor suppressor and oncogene: The WT1 story. Leukemia 2007, 21, 868-876. [CrossRef] [PubMed]

23. Schmid, D.A.; Irving, M.B.; Posevitz, V.; Hebeisen, M.; Posevitz-Fejfar, A.; Sarria, J.C.; Gomez-Eerland, R.; Thome, M.; Schumacher, T.N.; Romero, P.; et al. Evidence for a TCR affinity threshold delimiting maximal CD8 T cell function. J. Immunol. 2010, 184, 4936-4946. [CrossRef] [PubMed]

24. Henderson, R.A.; Michel, H.; Sakaguchi, K.; Shabanowitz, J.; Appella, E.; Hunt, D.F.; Engelhard, V.H. HLA-A2.1-associated peptides from a mutant cell line: A second pathway of antigen presentation. Science 1992, 255, 1264-1266. [CrossRef] [PubMed]

25. Nilsson, K.; Bennich, H.; Johansson, S.G.; Ponten, J. Established immunoglobulin producing myeloma (IgE) and lymphoblastoid (IgG) cell lines from an IgE myeloma patient. Clin. Exp. Immunol. 1970, 7, 477-489. 
26. Britten, C.M.; Meyer, R.G.; Kreer, T.; Drexler, I.; Wolfel, T.; Herr, W. The use of HLA-A*0201-transfected K562 as standard antigen-presenting cells for CD8(+) T lymphocytes in IFN-gamma ELISPOT assays. J. Immunol. Methods 2002, 259, 95-110. [CrossRef]

27. Khan, M.; Carmona, S.; Sukhumalchandra, P.; Roszik, J.; Philips, A.; Perakis, A.A.; Kerros, C.; Zhang, M.; Qiao, N.; John, L.S.S.; et al. Cathepsin G Is Expressed by Acute Lymphoblastic Leukemia and Is a Potential Immunotherapeutic Target. Front. Immunol. 2017, 8, 1975. [CrossRef]

28. Campillo-Davo, D.; Fujiki, F.; Van den Bergh, J.M.; De Reu, H.; Smits, E.L.; Goosens, H.; Sugiyama, H.; Lion, E.; Berneman, Z.N.; Van Tendeloo, V.F. Efficient and non-genotoxic RNA-based engineering of human T cells using tumor-specific T cell receptors with minimal TCR mispairing. Front. Immunol. 2018, 9, 2503. [CrossRef]

29. Versteven, M.; Van den Bergh, J.M.J.; Broos, K.; Fujiki, F.; Campillo-Davo, D.; De Reu, H.; Morimoto, S.; Lecocq, Q.; Keyaerts, M.; Berneman, Z.; et al. A versatile T cell-based assay to assess therapeutic antigenspecific PD-1-targeted approaches. Oncotarget 2018, 9, 27797-27808. [CrossRef]

30. Hasan, A.N.; Selvakumar, A.; O'Reilly, R.J. Artificial Antigen Presenting Cells: An Off the Shelf Approach for Generation of Desirable T-Cell Populations for Broad Application of Adoptive Immunotherapy. Adv. Genet. Eng. 2015, 4, 130.

31. Bossi, G.; Gerry, A.B.; Paston, S.J.; Sutton, D.H.; Hassan, N.J.; Jakobsen, B.K. Examining the presentation of tumor-associated antigens on peptide-pulsed T2 cells. Oncoimmunology 2013, 2, e26840. [CrossRef]

32. Ritz, U.; Seliger, B. The transporter associated with antigen processing (TAP): Structural integrity, expression, function, and its clinical relevance. Mol. Med. 2001, 7, 149-158. [CrossRef] [PubMed]

33. Schweitzer, S.; Schneiders, A.M.; Langhans, B.; Kraas, W.; Jung, G.; Vidalin, O.; Inchauspe, G.; Sauerbruch, T.; Spengler, U. Flow cytometric analysis of peptide binding to major histocampatibility complex class I for hepatitis C virus core T-cell epitopes. Cytometry 2000, 41, 271-278. [CrossRef]

34. Luft, T.; Rizkalla, M.; Tai, T.Y.; Chen, Q.; MacFarlan, R.I.; Davis, I.D.; Maraskovsky, E.; Cebon, J. Exogenous peptides presented by transporter associated with antigen processing (TAP)-deficient and TAP-competent cells: Intracellular loading and kinetics of presentation. J. Immunol. 2001, 167, 2529-2537. [CrossRef]

35. Stanke, J.; Hoffmann, C.; Erben, U.; von Keyserling, H.; Stevanovic, S.; Cichon, G.; Schneider, A.; Kaufmann, A.M. A flow cytometry-based assay to assess minute frequencies of CD8+ T cells by their cytolytic function. J. Immunol. Methods 2010, 360, 56-65. [CrossRef] [PubMed]

36. Garrido, F.; Aptsiauri, N.; Doorduijn, E.M.; Garcia Lora, A.M.; van Hall, T. The urgent need to recover MHC class I in cancers for effective immunotherapy. Curr. Opin. Immunol. 2016, 39, 44-51. [CrossRef] [PubMed]

37. Leone, P.; Shin, E.C.; Perosa, F.; Vacca, A.; Dammacco, F.; Racanelli, V. MHC class I antigen processing and presenting machinery: Organization, function, and defects in tumor cells. J. Natl. Cancer Inst. 2013, 105, 1172-1187. [CrossRef]

38. Van Camp, K.; Cools, N.; Stein, B.; Van de Velde, A.; Goossens, H.; Berneman, Z.N.; Van Tendeloo, V. Efficient mRNA electroporation of peripheral blood mononuclear cells to detect memory $\mathrm{T}$ cell responses for immunomonitoring purposes. J. Immunol. Methods 2010, 354, 1-10. [CrossRef] [PubMed]

39. Britten, C.M.; Meyer, R.G.; Frankenberg, N.; Huber, C.; Wolfel, T. The use of clonal mRNA as an antigenic format for the detection of antigen-specific T lymphocytes in IFN-gamma ELISPOT assays. J. Immunol. Methods 2004, 287, 125-136. [CrossRef]

40. Etschel, J.K.; Huckelhoven, A.G.; Hofmann, C.; Zitzelsberger, K.; Maurer, K.; Bergmann, S.; Mueller-Schmucker, S.M.; Wittmann, J.; Spriewald, B.M.; Dorrie, J.; et al. HIV-1 mRNA electroporation of PBMC: A simple and efficient method to monitor T-cell responses against autologous HIV-1 in HIV-1-infected patients. J. Immunol. Methods 2012, 380, 40-55. [CrossRef]

41. Shao, H.; Zhang, W.; Hu, Q.; Wu, F.; Shen, H.; Huang, S. TCR mispairing in genetically modified T cells was detected by fluorescence resonance energy transfer. Mol. Biol. Rep. 2010, 37, 3951-3956. [CrossRef]

42. Al-Ramadi, B.K.; Jelonek, M.T.; Boyd, L.F.; Margulies, D.H.; Bothwell, A.L. Lack of strict correlation of functional sensitization with the apparent affinity of MHC/peptide complexes for the TCR. J. Immunol. 1995, 155, 662-673. [PubMed]

43. Echchakir, H.; Dorothee, G.; Vergnon, I.; Menez, J.; Chouaib, S.; Mami-Chouaib, F. Cytotoxic T lymphocytes directed against a tumor-specific mutated antigen display similar HLA tetramer binding but distinct functional avidity and tissue distribution. Proc. Natl. Acad. Sci. USA 2002, 99, 9358-9363. [CrossRef] [PubMed] 
44. Lawson, T.M.; Man, S.; Wang, E.C.; Williams, S.; Amos, N.; Gillespie, G.M.; Moss, P.A.; Borysiewicz, L.K. Functional differences between influenza A-specific cytotoxic T lymphocyte clones expressing dominant and subdominant TCR. Int. Immunol. 2001, 13, 1383-1390. [CrossRef] [PubMed]

45. Palermo, B.; Campanelli, R.; Mantovani, S.; Lantelme, E.; Manganoni, A.M.; Carella, G.; Da Prada, G.; della Cuna, G.R.; Romagne, F.; Gauthier, L.; et al. Diverse expansion potential and heterogeneous avidity in tumor-associated antigen-specific $\mathrm{T}$ lymphocytes from primary melanoma patients. Eur. J. Immunol. 2001, 31, 412-420. [CrossRef]

46. Bacher, P.; Scheffold, A. Flow-cytometric analysis of rare antigen-specific T cells. Cytom. A 2013, 83, 692-701. [CrossRef]

(C) 2020 by the authors. Licensee MDPI, Basel, Switzerland. This article is an open access article distributed under the terms and conditions of the Creative Commons Attribution (CC BY) license (http://creativecommons.org/licenses/by/4.0/). 